\title{
Downstream control of upstream open reading frames
}

\author{
Matthew S. Sachs ${ }^{1,2,6}$ and Adam P. Geballe ${ }^{3,4,5}$ \\ ${ }^{1}$ Department of Environmental and Biomolecular Systems, Oregon Health and Science University, Beaverton, Oregon 97006, \\ USA; ${ }^{2}$ Department of Molecular Microbiology and Immunology, Oregon Health and Science University, Portland, Oregon \\ 97201, USA; ${ }^{3}$ Divisions of Human Biology and Clinical Research, Fred Hutchinson Cancer Research Center, Seattle, \\ Washington 98109, USA; ${ }^{4}$ Department of Microbiology and Department of Medicine, University of Washington, \\ Seattle, Washington 98115, USA
}

Studies over the past two decades have uncovered a surprising variety of genetic regulatory mechanisms mediated by upstream AUG (uAUG) codons and their associated upstream open reading frames (uORFs) (Lovett and Rogers 1996; Geballe and Sachs 2000; Morris and Geballe 2000; Dever 2002; Vilela and McCarthy 2003). As illustrated by an intriguing report by Mehta et al. (2006) in this issue of Genes \& Development, we still have much to learn about the array of cis-acting sequences, trans-acting factors, and specific mechanisms that act with uORFs to regulate protein synthesis. Mehta et al. discovered that sequences in the $3^{\prime}$ UTR of the Her-2 oncogene overcome the negative impact of the UORF on gene expression in some types of tumor cells. In this perspective, we provide a current view of uORF functions and regulatory mechanisms, highlighting the implications of the results of Mehta et al. (2006) for understanding ubiquitous but still-mysterious events occurring during translation and implications for tumor cell biology.

\section{Unexpected role for the 3'UTR in a uORF regulatory mechanism}

The observation that a large number of genes involved in cellular growth regulation, including the Her-2 oncogene, express mRNAs with uORFs led to speculations that uORF-mediated regulatory mechanisms might be important to prevent uncontrolled cell growth. The Her-2 gene is amplified and the protein is overexpressed in a variety of cancers, including $20 \%-30 \%$ of breast cancers. In such cases, the overexpression of Her- 2 contributes to the malignant phenotype and therapies directed against Her-2 are useful for treating these tumors (for review, see Rabindran 2005). Thus, understanding

Correspondence.

${ }^{5}$ E-MAIL ageballe@fhcrc.org; FAX (206) 667-6523.

${ }^{6}$ E-MAIL msachs@ebs.ogi.edu; FAX (503) 748-1464.

Article and publication are at http://www.genesdev.org/cgi/doi/10.1101/ gad.1427006. how Her-2 expression is controlled is of considerable clinical importance.

Previous studies had revealed that the well-conserved uORF in Her-2 controlled its expression at the level of translation (Child et al. 1999a,b). When fused to a reporter gene, the uORF inhibits downstream translation in multiple cell types. However, the Her-2 mRNA is translated much more efficiently in some cancer cell lines compared with normal cells, as assessed by loading of the mRNA onto larger polysomes, even though the UORF is present on the mRNA in both types of cells (Child et al. 1999a). In further investigations of the basis for this cell type specificity of Her-2 mRNA translation, Mehta et al. (2006) now report the surprising discovery, based on analyses of the levels of expression of a reporter gene enzyme and mRNA, that the Her-2 mRNA 3'UTR is able to eliminate the inhibitory effect of the UORF in a cell-type-specific manner. The effect of the 3'UTR to stimulate translation in some cells appears to be specific for ameliorating uORF-mediated inhibition of translation of Her-2. It does not increase the already high level expression from an mRNA lacking the uORF, so it is not a general enhancer of translation. Interestingly, it also does not increase translation that is inhibited by a stable stem-loop positioned at the $5^{\prime}$ end of the mRNA. Nothing from past studies of UORFs predicts such a role for 3'UTR sequences in affecting a UORF regulatory mechanism. Before considering how such a 3'UTR element might act, we first review recent analyses of the prevalence of uORFs and discuss mechanisms by which uORFs have been shown to affect gene expression.

\section{Prevalence of uORFs in eukaryotic mRNAs}

We define a "uAUG" as any codon used by ribosomes to initiate protein synthesis that is present in an mRNA 5' leader (also called 5'UTR), upstream of the initiation codon used by ribosomes to produce the principal protein product. The reading frame downstream from a uAUG is its associated "uORF." We refer to the principal protein initiation site as the "pAUG" codon. 
Although these definitions seem relatively simple, there are subtleties. First, although AUG codons constitute the predominant codon used for translation initiation, non-AUG codons initiate translation in some cases, and upstream non-AUG initiators have the potential to affect translation from the pAUG codon by the same mechanisms as conventional uAUGs. At least one uORF initiates at a CUG codon (Warnakulasuriyarachchi et al. 2003). Since there is no reliable way to predict non-AUG codons that initiate translation of uORFs by sequence analysis, they might be more common than is currently recognized. Second, we consider the reading frame initiating at a uAUG to be a uORF regardless of its length or the position of its termination codon relative to the pAUG codon. Therefore, the uORF may overlap with the downstream principal ORF or with other uORFs. The spectrum of regulatory events that affect uORF function can depend on the spatial relationship of its initiation codon and stop codon relative to these other reading frames. The special case in which a uAUG codon starts a reading frame that fused in-frame with the principal ORF and therefore is translated to produce a polypeptide extended at its $\mathrm{N}$ terminus is not a distinct uORF, but differential start-codon selection in such cases may involve mechanisms similar to those that contribute to the use of other uAUGs.

How common are uAUGs and uORFs? Attempts to use genomic DNA sequence information to identify uAUG codons and uORFs have been confounded by obstacles including the requirement for knowing the authentic $5^{\prime}$ ends of the mRNA and the correct pAUG codons. Mapping 5' ends of specific mRNAs is a laborintensive process that has been accomplished only for a small fraction of genes annotated in sequence databases. Alternative promoter usage and/or alternative splicing can remove uORFs that may be present in some mRNAs (Brown et al. 1999; Colot et al. 2005; Law et al. 2005; de Almeida et al. 2006). Methods such as "oligo-capping" and "vector-capping" may overcome some of these obstacles by enabling high-throughput construction and analyses of cDNA libraries in which cDNA sequences that extend to the cap site can be discerned (Suzuki et al. 2000; Kato et al. 2005).

Early compilations of mammalian genes with known mRNA leader sequences revealed that uORFs are present in $10 \%$ of mRNAs and are particularly common in transcripts for oncogenes, growth factors, and cellular receptors (Kozak 1987a). Recent genome-wide analyses of mammalian cDNAs have affirmed the conclusion that uORFs are common (Churbanov et al. 2005; Iacono et al. 2005). AUG triplets are less frequent in 5'UTRs than other nucleotide triplets but are nonetheless present in $15 \%-53 \%$ of mRNAs, depending on the organism. Analyses of human genes represented in an "oligocapped" database revealed that $\sim 30 \%$ contain one or more uORFs (Suzuki et al. 2000).

Although uAUG codons are statistically underrepresented in 5'UTRs, when present, they are the most highly conserved individual codon in the 5'UTRs in comparisons of orthologous genes of human and mouse, or rat and mouse (Churbanov et al. 2005). The Her-2 $\mathrm{UORF}$ is well conserved in sequence and position in these three species (Child et al. 1999a). Analyses of fungal genomes indicate that uORFs are also common in these organisms and that a substantial subset of them is evolutionarily conserved (Galagan et al. 2005; Zhang and Dietrich 2005).

Several provocative findings have emerged from in silico analyses of uAUG codons. First, conserved uORFs tend to be shorter than random ORFs, possibly related to the negative association of uORF length and reinitiation potential (see below). Second, the presence of uAUG codons has been correlated with a weaker context of the pAUG codon (Rogozin et al. 2001). One interpretation of this observation is that these mRNAs encode proteins that are harmful when abundant and the combination of inhibitory uORFs and weak pAUG codons provides redundant controls, ensuring a low level of translation from the pAUG.

\section{uORF barrier function}

The scanning model of eukaryotic translation initiation holds that the $40 S$ ribosomal subunit with its associated factors, including the ternary complex eIF2.GTP.MettRNA $_{i}^{\text {Met }}$, loads on to an mRNA at the cap, then scans in a $3^{\prime}$ direction until it encounters an initiation codon, at which point the $60 \mathrm{~S}$ ribosomal subunit joins and protein synthesis begins. Thus, uORFs interpose a barrier predicted to prevent ribosomal access to the downstream pAUG.

Several well-documented mechanisms enable expression of the downstream ORF despite the presence of a uORF. First, some or all ribosomes may simply bypass the uAUG codon. The context of surrounding nucleotides is one clear determinant of AUG codon recognition by the ribosome. The most common bases surrounding AUG codons ([A/G]CC aug G) are also the ones that make the strongest initiation sites and, when surrounding a uAUG codon, they are the most likely ones to engage the scanning ribosomes, provided they are not too close to the mRNA 5' cap (Kozak 1991, 1999). However, many uAUG codons do not have the fully optimal sequence (Suzuki et al. 2000), leading to the prediction that at least some scanning ribosomes are likely to bypass them, a process known as leaky scanning. The Her-2 uORF has a very strong context AUG codon, and most scanning ribosomes likely initiate at this site (Child et al. 1999b; Mehta et al. 2006). In addition to leaky scanning, the uAUG codon may, in principle, be bypassed by mechanisms of ribosomal hopping or shunting (Yueh and Schneider 1996; Ryabova and Hohn 2000; Rogers et al. 2004), or because of the presence of sequences defined as internal ribosomal entry sites (IRESs) (Komar and Hatzoglou 2005; Kozak 2005).

Even if the ribosome recognizes a uAUG codon and translates the uORF, it might reinitiate at a downstream AUG codon. The efficiency of reinitiation varies depending on parameters including the length of the uORF (Rajkowitsch et al. 2004). Reinitiation tends to be less effi- 
cient after translation of longer uORFs compared with shorter ones. Events during and immediately after translation termination also seem to have major influence on the ribosome's reinitiation potential (Grant and Hinnebusch 1994). The basis for these phenomena have not been elucidated but may result from changes in the ribosomes or associated factors that are needed for efficient reinitiation but are lost during elongation and/or by events occurring during termination that are influenced by the context of nucleotides surrounding the termination codon and by factors directly or indirectly associated with the mRNA. The nature of these factors is unknown.

Another key feature that can impact reinitiation is the intercistronic spacing between the uORF termination codon and the downstream AUG at which translation reinitiates (Kozak 1987b; Grant et al. 1994). At a minimum, a ribosome that has translated a uORF needs to reacquire the initiator Met-tRNA $\mathrm{i}_{\mathrm{i}}^{\text {Met }}$ before it can restart synthesizing protein. This recharging of the ribosome presumably occurs while it is traversing the intercistronic region. Thus, longer intercistronic distances tend to allow more efficient reinitiation. The short length of the Her-2 uORF (six codons) and the short intercistronic region (5 nucleotides [nt]) might, therefore, be predicted to have opposite effects on the reinitiation efficiency of ribosomes that have translated the uORF. Experimental data suggest that at least some ribosomes reinitiate at the Her-2 pAUG codon (Child et al. 1999b; Mehta et al. 2006).

Given these considerations, a uORF might simply impose a constitutive barrier to the scanning ribosome and thereby reduce the number of ribosomes that, by leaky scanning or reinitiation mechanisms, gain access to the pAUG codon. However, the effects of the uORF may be regulated by specific conditions, such as the availability of translation initiation factor eIF2 (see below) or, as appears to be the case for Her-2, by factors in specific cell types that function in concert with the mRNA 3'UTR. Our current inability to predict a priori conditions that alter uORF function means that uORFs that appear to be constitutive inhibitors may turn out to be regulated by conditions that have not yet been identified. We next consider a few examples of the more complex ways that uORFs are known to affect gene expression.

\section{Sequence-dependent uORFs}

Stalling at a rare codon in a uORF can strongly impact downstream translation, even if initiation at the UORF is not efficient (Meijer and Thomas 2003). In other cases, the specific codons used in UORF coding sequences are not important, but the sequence of the nascent peptide specified by the uORF is the key to its regulatory effects. In these cases, the inhibitory effect on downstream translation is eliminated by at least some missense but not by synonymous mutations within the uORF. The nascent peptide products specified by these uORFs cause ribosomes that are translating them to stall. In the case of the human cytomegalovirus UL4 gene, ribosomes that have translated the uORF stall at the termination codon in a complex that includes eukaryotic release factor 1 (eRF1) but not eRF3 (Janzen et al. 2002). The fungal arginine attenuator peptide (AAP), produced by translation of an evolutionarily conserved UORF, can stall ribosomes engaged in either elongation or termination in response to a high concentration of arginine (Wang et al. 1999; Fang et al. 2000). A six-codon sequence-dependent uORF in the mammalian gene specifying $S$-adenosylmethionine decarboxylase causes ribosomes to stall at the $\mathrm{UORF}$ termination codon in the presence of polyamines (Law et al. 2001; Raney et al. 2002). In these cases, uORFmediated ribosome stalling acts as a blockade to reduce the accessibility of the pAUG to scanning ribosomes. For the AAP, ribosome stalling at the UORF termination codon also triggers nonsense-mediated mRNA decay (NMD) (Gaba et al. 2005). Thus the translational effect of this UORF has additional consequences for mRNA stability. Nascent prokaryotic peptides specified by uORFs (called leader peptides) also can stall ribosomes in response to small molecules (Lovett 1994; Gong and Yanofsky 2002; Cruz-Vera et al. 2005; Mankin 2006). The existence of known sequence-dependent uORFs in such diverse systems suggests that there are many more examples awaiting discovery.

\section{Regulated reinitiation}

Translational control of the Saccharomyces cerevisiae GNC4 gene is one of the best characterized uORF-mediated regulatory mechanisms (Hinnebusch 2005). The GCN4 mRNA, which encodes a transcriptional activator of amino acid biosynthetic genes (and other genes as well), contains four short uORFs. Independent of amino acid availability, most ribosomes translate the first uORF. When amino acid levels are high, they reacquire eIF2.GTP.Met-tRNA ${ }_{i}^{\text {Met }}$ relatively rapidly afterward and preferentially reinitiate at another UORF, after which they appear unable to reinitiate again. The presence of deacylated tRNA, as occurs during amino acid limitation, activates the eIF $2 \alpha$ kinase Gcn2p. As a result, ternary complex levels are low, and ribosomes are unable to reinitiate for a longer period, after which they are likely to have bypassed the other inhibitory uORFs and reached the Gcn $4 p$ initiation codon. A still poorly understood feature of GCN4 regulation concerns why ribosomes can reinitiate after translation of uORF1 but not other uORFs. This difference depends in part on cis-acting sequences, the final codon (or penultimate) codon, and the nucleotides immediately downstream from uORF1. A similar mechanism has been shown to control expression of the mammalian ATF4 gene, a mediator of the unfolded protein stress response (Lu et al. 2004; Vattem and Wek 2004). In this case, the kinase PERK (or PEK) is responsible for phosphorylating eIF $2 \alpha$ and limiting the availability of the ternary complex. The control of mammalian CD36 translation by glucose also involves reinitiation following uORF translation, but the mechanism remains to be elucidated (Griffin et al. 2001).

GCN4 illustrates how a uORF can differentially guide 
a ribosome to alternative downstream AUG codons. In the case of GCN4, the alternative sites are another uAUG or the pAUG. However, in other mRNAs in which there is only one uAUG codon, the same mechanism could influence whether a ribosome initiates at the pAUG codon or at an AUG codon further downstream that may be in-frame within the principal ORF and thus lead to synthesis of an $\mathrm{N}$-terminally truncated protein. This sort of regulatory scheme occurs in the case of the CAAT enhancer-binding proteins $\alpha$ and $\beta$ (Calkhoven et al. 2000), and the SCL protein (Calkhoven et al. 2003) and may be germane to the Her-2 uORF mechanism (Child et al. 1999b).

\section{Effects on RNA structure}

Prokaryotic ribosomes do not reach initiation codons by scanning, but bind directly to them as a result of pairing of a nearby Shine-Delgarno sequence in the mRNA with rRNA (Laursen et al. 2005). Thus, prokaryotic uORFs do not affect downstream translation initiation by controlling scanning. However, several examples of translational control in prokaryotes mediated by short uORFs are instructive for understanding eukaryotic uORFs. For example, translation of uORFs (leader peptides) in the cat (chloramphenicol acetyl transferase) and erm C (a ribosome methylase conferring resistance to macrolide antibiotics) mRNAs, in the presence of antibiotic cofactors, stalls ribosomal transit and unmasks a downstream Shine-Delgarno sequence, thereby inducing expression of the downstream antibiotic-resistance gene (Lovett and Rogers 1996). In a conceptually similar manner, translation of a uORF in the eukaryotic cat-1 (cationic amino acid transporter-1) gene can alter mRNA structure to unmask an IRES element, activating CAT-1 synthesis. Phosphorylation of eIF $2 \alpha$ or introduction of rare codons into the UORF activate the IRES (Yaman et al. 2003; Fernandez et al. 2005). Thus uORFs have the potential to affect local RNA structure and could thereby affect translation or other events in RNA biology.

\section{3'UTR element regulation of uORF activity}

Prior to the report of Mehta et al. (2006), cis-acting sequence elements known to affect uORF function were all within or near the uORF. Thus, the detection of a role for 3'UTR sequences in the case of the Her-2 uORF regulatory mechanism is an unexpected discovery that needs to be integrated into our concepts of translational regulation by uORFs.

Given their presence in the same transcript, how might a 3'UTR sequence overcome a UORF inhibitory effect? In theory, each of the steps required for inhibition by a UORF could be counteracted by a regulatory element. Such a regulator could promote bypass of the uAUG codon (Fig. 1, arrow 1). However, Mehta et al. (2006) provide evidence that this is not the mechanism of the Her-2 3'UTR element, since initiation at the uAUG increases approximately twofold upon addition of

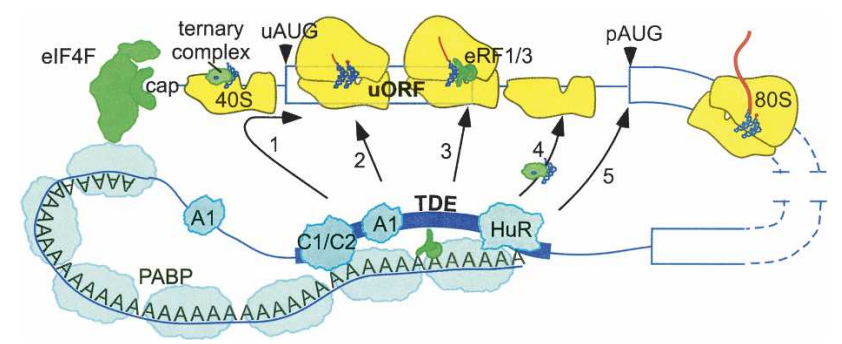

Figure 1. Potential mechanisms of $3^{\prime}$ UTR sequence regulation of UORF inhibitory activity. A $3^{\prime}$ UTR sequence that alleviates the inhibitory effects of uORFs could, in principle, act at one or more of several steps. It could promote leaky scanning past the uAUG codon (arrow 1) or eliminate an elongation block during uORF translation (arrow 2). It also could alter the efficiency of termination (arrow 3), of recharging the 40S with eIF2-GTP.Met-tRNA ${ }_{i}^{\text {Met }}$ (ternary complex) or other initiation factors (arrow 4), or of pAUG codon recognition (arrow 5). Proteins such as HnRNPC1/C2 $(\mathrm{C} 1 / \mathrm{C} 2)$ and HuR that bind specifically to the $3^{\prime}$ TDE in the Her-2 $3^{\prime}$ UTR may mediate the derepression mechanism through their direct or indirect interactions with other 3'UTR-binding factors such as PABP, hnRNPA1 (A1), and eukaryotic release factors 1 and 3 (eRF1/3). The illustration is not to scale; for example, the entire Her-2 uORF coding region could be covered by only one ribosome.

the $3^{\prime}$ UTR sequences. Another possible effect of the 3' element could be to alter inhibitory events occurring during the elongation phase or prior to completing the termination phase of uORF translation, for example, by reducing stalling caused by a rare codon or the nascent peptide. These possibilities are unlikely to explain the Her-2 3'UTR effect because Mehta et al. found that the inhibitory effect of a uORF in the Ship-2 5'UTR (which has no obvious sequence similarity to the Her-2 uORF) is also derepressed by the Her-2 3'UTR element, suggesting that the mechanism does not require specific coding sequence of the Her-2 uORF (Fig. 1, arrow 2). The significance, if any, of the conservation of the Her-2 uORF peptide sequence among mammalian species remains to be determined.

The more likely possibilities are therefore that the $3^{\prime}$ element acts by altering the specific events that occur during (Fig. 1, arrow 3) or after (Fig. 1,arrow 4) termination of UORF translation and that these changes impact the ability of the ribosome to reinitiate at the pAUG codon. Alternatively, the 3'UTR element might act directly at the pAUG codon to stimulate its recognition (Fig. 1, arrow 5). It is also conceivable that effects on termination of translation of the principal ORF might alter the translational apparatus to increase reinitiation at the pAUG.

Mehta et al. (2006) mapped essential regulatory sequences within the Her-2 3'UTR to nucleotides immediately downstream from the termination codon of the major ORF and to a 73-nt segment, termed the translational derepression element (TDE), closer to the 3 ' end of the mRNA. The requirement for the nucleotides immediately following the termination codon was not investigated further but should be of future interest because, 
at least for GCN4 uORF1, the region following the termination codon is crucial for enabling ribosomes to subsequently reinitiate, and thus these sequences in the 3'UTR of Her-2 might contribute to efficient recycling of reinitiation-competent ribosomes from the $3^{\prime} \mathrm{UTR}$ to the 5'UTR.

The TDE was examined in more detail. Two RNAbinding proteins, $\mathrm{HuR}$ and $\mathrm{HnRNPC} / \mathrm{C} 2$, bind specifically to the TDE. HnRNP A1 binds the TDE too, but it also binds to other RNA sequences in the Her-2 $3^{\prime}$ UTR. Poly(A)-binding protein (PABP) apparently binds to HuR and HnRNPC1/C2 based on their coimmunoprecipitation even after RNase treatment. The authors postulate that a complex of proteins that bind the TDE mediate the derepression mechanism. In support of this hypothesis, the abundance of TDE-binding activity appears to be greater in tumor cells that express high levels of Her- 2 and in which the $3^{\prime}$ UTR is best able to counteract the inhibitory effect of the uORF. However, the overall abundance of these proteins may not be a reliable indicator of TDE-binding activity since their subcellular distribution among cell types may differ, as demonstrated for HuR (Barreau et al. 2006; Mehta et al. 2006). It will be very interesting to determine whether these 3'UTR-binding proteins or other factors (possibly including microRNAs) that can bind to the Her-2 3'UTR are responsible for controlling the efficient translation of the Her-2 mRNA in tumor cells. Interfering with the factors that stimulate translation might provide a new therapeutic approach for cancers with Her-2 overexpression.

It is clear from many previous studies that factors binding to the 3'UTR considerably influence gene expression (see Kuersten and Goodwin 2003; Wilkie et al. 2003). In some cases, these elements increase mRNA polyadenylation, thereby increasing gene expression. Factors that bind to 3'UTRs can result in localization of translation to specific cellular locations. Often 3'UTRbinding factors can control the stability of the mRNA; this is the case for HuR (Barreau et al. 2005), which binds to the Her-2 TDE. When factors that bind the 3'UTR have direct effects on translation, they are typically inhibitory (Beckmann et al. 2005 and references therein), although HuR can have a stimulatory effect (for review, see Espel 2005). Even with the delineation of key cisacting sequences in the $3^{\prime}$ UTR and the characterization of possible trans-acting protein factors, the mechanism by which the 3 'UTR counteracts uORF-mediated inhibition of translation remains to be clarified. The discovery that PABP stimulates translation initiation and binds both to the poly(A) tail and eIF-4G, a component of the 5 '-cap-binding complex, gave rise to the "closed-loop model" of translation (Kahvejian et al. 2005 and references therein). According to this model, the $5^{\prime}$ and $3^{\prime}$ ends of an mRNA are tethered by interacting proteins, and these interactions could stimulate initiation in several independent ways. Initiation itself could be stimulated by the PABP-eIF4G interaction, and the closed loop could additionally allow ribosomes that have completed translation and are transiting the 3'UTR to be recycled to the $5^{\prime}$ region of the mRNA. Although the closed-loop model does not readily explain how the Her-2 3'UTR specifically overcomes the inhibitory effects of a UORF, it does provide the useful concept that 3'UTRs may influence events at the $5^{\prime}$ UTR as a result of their spatial proximity.

Any model of the Her-2 3'UTR regulatory effects needs to account for the observation that the element mitigates the inhibitory effects of a uORF but not a stem-loop in the 5'UTR that blocks access of scanning ribosomes to the pAUG. One possibility that would be consistent with the data is that factors binding to the 3'UTR element enhance the delivery of other factors to the ribosomes translating the uORF. Such factors might not significantly enhance the primary initiation event at the uAUG, but might significantly increase the capacity for reinitiation following uORF translation. For example, TDE-binding proteins interact with $\mathrm{PABP}$, which, in turn, interacts with eRF3; such a complex might alter termination of uORF translation to stimulate subsequent reinitiation (Fig. 1, arrow 3). Alternatively, the TDE, in the presence of cell-specific factors and in the context of a native $5^{\prime} \mathrm{UTR}$, might generally promote initiation in the $5^{\prime} \mathrm{UTR}$; initiation at the uORF might already be very efficient, and thus not benefit greatly from this stimulation, while the reinitiation event, which is suboptimal, would see greater relative stimulation. More work will need to be done to distinguish among these and other possibilities.

\section{Conclusion}

The intriguing report from Mehta et al. (2006) provides important new insights into uORF-mediated regulatory mechanisms. Most striking is the finding that in the absence of the Her-2 3'UTR, the Her-2 uORF is inhibitory in all cell types tested, but the $3^{\prime}$ UTR relieves inhibition in specific tumor cells. The Her-2 3'UTR counteracts the inhibitory effect of two different inhibitory uORFs but not an inhibitory stem-loop. Future work more precisely delineating the relevant cis-acting sequences in the 5'UTR and 3'UTRs and determining which of the various candidate trans-acting factors are essential will help elucidate the underlying mechanism of Her-2 overexpression in cancer cells. The possibility that the stimulatory effect of the Her-2 $3^{\prime} \mathrm{UTR}$ is clinically relevant in tumors expressing high levels of Her-2, and that interfering with $3^{\prime}$ UTR could be a clinically relevant approach will also be important to consider. Since recent bioinformatics analyses suggest that uORFs are remarkably common, the report of Mehta et al. should stimulate and guide research into uORF function in many other systems.

\section{Acknowledgments}

This work was supported by Public Health Service grants AI26672 (to A.P.G.) and GM47498 (to M.S.S.). 


\section{References}

Barreau, C., Paillard, L., and Osborne, H.B. 2005. AU-rich elements and associated factors: Are there unifying principles? Nucleic Acids Res. 33: 7138-7150.

Beckmann, K., Grskovic, M., Gebauer, F., and Hentze, M.W. 2005. A dual inhibitory mechanism restricts msl-2 mRNA translation for dosage compensation in Drosophila. Cell 122: 529-540.

Brown, C.Y., Mize, G.J., Pineda, M., George, D.L., and Morris, D.R. 1999. Role of two upstream open reading frames in the translational control of oncogene $m d m 2$. Oncogene 18: 5631-5637.

Calkhoven, C.F., Muller, C., and Leutz, A. 2000. Translational control of $\mathrm{C} / \mathrm{EBP} \alpha$ and $\mathrm{C} / \mathrm{EBP} \beta$ isoform expression. Genes \& Dev. 14: 1920-1932.

Calkhoven, C.F., Muller, C., Martin, R., Krosl, G., Hoang, T., and Leutz, A. 2003. Translational control of SCL-isoform expression in hematopoietic lineage choice. Genes \& Dev. 17: 959-964.

Child, S.J., Miller, M.K., and Geballe, A.P. 1999a. Cell typedependent and -independent control of HER-2/neu translation. Int. J. Biochem. Cell Biol. 31: 201-213.

. 1999b. Translational control by an upstream open reading frame in the HER-2/neu transcript. J. Biol. Chem. 274: 24335-24341.

Churbanov, A., Rogozin, I.B., Babenko, V.N., Ali, H., and Koonin, E.V. 2005. Evolutionary conservation suggests a regulatory function of AUG triplets in $5^{\prime}$-UTRs of eukaryotic genes. Nucleic Acids Res. 33: 5512-5520.

Colot, H.V., Loros, J.J., and Dunlap, J.C. 2005. Temperaturemodulated alternative splicing and promoter use in the circadian clock gene frequency. Mol. Biol. Cell 16: 5563-5571.

Cruz-Vera, L.R., Rajagopal, S., Squires, C., and Yanofsky, C. 2005. Features of ribosome-peptidyl-tRNA interactions essential for tryptophan induction of tha operon expression. Mol. Cell 19: 333-343.

de Almeida, R.A., Heuser, T., Blaschke, R., Bartram, C.R., and Janssen, J.W. 2006. Control of MYEOV protein synthesis by upstream open reading frames. J. Biol. Chem. 281: 695-704.

Dever, T.E. 2002. Gene-specific regulation by general translation factors. Cell 108: 545-556.

Espel, E. 2005. The role of the AU-rich elements of mRNAs in controlling translation. Semin. Cell Dev. Biol. 16: 59-67.

Fang, P., Wang, Z., and Sachs, M.S. 2000. Evolutionarily conserved features of the arginine attenuator peptide provide the necessary requirements for its function in translational regulation. J. Biol. Chem. 275: 26710-26719.

Fernandez, J., Yaman, I., Huang, C., Liu, H., Lopez, A.B., Komar, A.A., Caprara, M.G., Merrick, W.C., Snider, M.D., Kaufman, R.J., et al. 2005. Ribosome stalling regulates IRES-mediated translation in eukaryotes, a parallel to prokaryotic attenuation. Mol. Cell 17: 405-416.

Gaba, A., Jacobson, A., and Sachs, M.S. 2005. Ribosome occupancy of the yeast CPA1 upstream open reading frame termination codon modulates nonsense-mediated mRNA decay. Mol. Cell 20: 449-460.

Galagan, J.E., Calvo, S.E., Cuomo, C., Ma, L.J., Wortman, J.R., Batzoglou, S., Lee, S.I., Basturkmen, M., Spevak, C.C., Clutterbuck, J., et al. 2005. Sequencing of Aspergillus nidulans and comparative analysis with $A$. fumigatus and $A$. oryzae. Nature 438: 1105-1115.

Geballe, A.P. and Sachs, M.S. 2000. Translational control by upstream open reading frames. In Translational control of gene expression (eds. N. Sonenberg et al.), pp. 595-614. Cold Spring Harbor Laboratory Press, Cold Spring Harbor, NY.
Gong, F. and Yanofsky, C. 2002. Instruction of the translating ribosome by nascent peptide. Science 297: 1864-1867.

Grant, C.M. and Hinnebusch, A.G. 1994. Effect of sequence context at stop codons on efficiency of reinitiation in GCN4 translational control. Mol. Cell. Biol. 14: 606-618.

Grant, C.M., Miller, P.F., and Hinnebusch, A.G. 1994. Requirements for intercistronic distance and level of eukaryotic initiation factor 2 activity in reinitiation on GCN4 mRNA vary with the downstream cistron. Mol. Cell. Biol. 14: 2616-2628.

Griffin, E., Re, A., Hamel, N., Fu, C., Bush, H., McCaffrey, T., and Asch, A.S. 2001. A link between diabetes and atherosclerosis: Glucose regulates expression of CD36 at the level of translation. Nat. Med. 7: 840-846.

Hinnebusch, A.G. 2005. Translational regulation of GCN4 and the general amino acid control of yeast. Annu. Rev. Microbiol. 59: 407-450.

Iacono, M., Mignone, F., and Pesole, G. 2005. uAUG and uORFs in human and rodent $5^{\prime}$ untranslated mRNAs. Gene 349: 97-105.

Janzen, D.M., Frolova, L., and Geballe, A.P. 2002. Inhibition of translation termination mediated by an interaction of eukaryotic release factor 1 with a nascent peptidyl-tRNA. Mol. Cell. Biol. 22: 8562-8570.

Kahvejian, A., Svitkin, Y.V., Sukarieh, R., M'Boutchou, M.N., and Sonenberg, N. 2005. Mammalian poly(A)-binding protein is a eukaryotic translation initiation factor, which acts via multiple mechanisms. Genes \& Dev. 19: 104-113.

Kato, S., Ohtoko, K., Ohtake, H., and Kimura, T. 2005. Vectorcapping: A simple method for preparing a high-quality fulllength cDNA library. DNA Res. 12: 53-62.

Komar, A.A. and Hatzoglou, M. 2005. Internal ribosome entry sites in cellular mRNAs: The mystery of their existence. $J$. Biol. Chem. 280: 23425-23428.

Kozak, M. 1987a. An analysis of 5'-noncoding sequences from 699 vertebrate messenger RNAs. Nucleic Acids Res. 15: 8125-8148.

- 1987b. Effects of intercistronic length on the efficiency of reinitiation by eucaryotic ribosomes. Mol. Cell. Biol. 7: $3438-3445$.

-1991. A short leader sequence impairs the fidelity of initiation by eukaryotic ribosomes. Gene Expr. 1: 111-115.

- 1999. Initiation of translation in prokaryotes and eukaryotes. Gene 234: 187-208.

. 2005. A second look at cellular mRNA sequences said to function as internal ribosome entry sites. Nucleic Acids Res. 33: 6593-6602.

Kuersten, S. and Goodwin, E.B. 2003. The power of the 3' UTR: Translational control and development. Nat. Rev. Genet. 4: 626-637.

Laursen, B.S., Sorensen, H.P., Mortensen, K.K., and SperlingPetersen, H.U. 2005. Initiation of protein synthesis in bacteria. Microbiol. Mol. Biol. Rev. 69: 101-123.

Law, G.L., Raney, A., Heusner, C., and Morris, D.R. 2001. Polyamine regulation of ribosome pausing at the upstream open reading frame of $S$-adenosylmethionine decarboxylase. $I$. Biol. Chem. 276: 38036-38043.

Law, G.L., Bickel, K.S., MacKay, V.L., and Morris, D.R. 2005. The undertranslated transcriptome reveals widespread translational silencing by alternative $5^{\prime}$ transcript leaders. Genome Biol. 6: R111.

Lovett, P.S. 1994. Nascent peptide regulation of translation. J. Bacteriol. 176: 6415-6417.

Lovett, P.S. and Rogers, E.J. 1996. Ribosome regulation by the nascent peptide. Microbiol. Rev. 60: 366-385.

Lu, P.D., Harding, H.P., and Ron, D. 2004. Translation reinitia- 
tion at alternative open reading frames regulates gene expression in an integrated stress response. J. Cell Biol. 167: 27-33.

Mankin, A.S. 2006. Nascent peptide in the "birth canal" of the ribosome. Trends Biochem. Sci. 31: 11-13.

Mehta, A., Trotta, C., and Peltz, S. 2006. Derepression of the Her-2 uORF is mediated by a novel post-transcriptional control mechanism in cancer cells. Genes \& Dev. (this issue).

Meijer, H.A. and Thomas, A.A. 2003. Ribosomes stalling on uORF1 in the Xenopus Cx41 5' UTR inhibit downstream translation initiation. Nucleic Acids Res. 31: 3174-3184.

Morris, D.R. and Geballe, A.P. 2000. Upstream open reading frames as regulators of mRNA translation. Mol. Cell. Biol. 20: $8635-8642$.

Rabindran, S.K. 2005. Antitumor activity of HER-2 inhibitors. Cancer Lett. 227: 9-23.

Rajkowitsch, L., Vilela, C., Berthelot, K., Ramirez, C.V., and McCarthy, J.E. 2004. Reinitiation and recycling are distinct processes occurring downstream of translation termination in yeast. J. Mol. Biol. 335: 71-85.

Raney, A., Law, G.L., Mize, G.J., and Morris, D.R. 2002. Regulated translation termination at the upstream open reading frame in $S$-adenosylmethionine decarboxylase mRNA. I. Biol. Chem. 277: 5988-5994.

Rogers Jr., G.W., Edelman, G.M., and Mauro, V.P. 2004. Differential utilization of upstream AUGs in the $\beta$-secretase mRNA suggests that a shunting mechanism regulates translation. Proc. Natl. Acad. Sci. 101: 2794-2799.

Rogozin, I.B., Kochetov, A.V., Kondrashov, F.A., Koonin, E.V., and Milanesi, L. 2001. Presence of ATG triplets in $5^{\prime}$ untranslated regions of eukaryotic cDNAs correlates with a 'weak' context of the start codon. Bioinformatics 17: 890900.

Ryabova, L.A. and Hohn, T. 2000. Ribosome shunting in the cauliflower mosaic virus 35S RNA leader is a special case of reinitiation of translation functioning in plant and animal systems. Genes \& Dev. 14: 817-829.

Suzuki, Y., Ishihara, D., Sasaki, M., Nakagawa, H., Hata, H., Tsunoda, T., Watanabe, M., Komatsu, T., Ota, T., Isogai, T., et al. 2000. Statistical analysis of the $5^{\prime}$ untranslated region of human mRNA using 'Oligo-Capped' cDNA libraries. Genomics 64: 286-297.

Vattem, K.M. and Wek, R.C. 2004. Reinitiation involving upstream ORFs regulates ATF4 mRNA translation in mammalian cells. Proc. Nat1. Acad. Sci. 101: 11269-11274.

Vilela, C. and McCarthy, J.E. 2003. Regulation of fungal gene expression via short open reading frames in the mRNA $5^{\prime}$ untranslated region. Mol. Microbiol. 49: 859-867.

Wang, Z., Gaba, A., and Sachs, M.S. 1999. A highly conserved mechanism of regulated ribosome stalling mediated by fungal arginine attenuator peptides that appears independent of the charging status of arginyl-tRNAs. J. Biol. Chem. 274: 37565-37574.

Warnakulasuriyarachchi, D., Ungureanu, N.H., and Holcik, M. 2003. The translation of an antiapoptotic protein HIAP2 is regulated by an upstream open reading frame. Cell Death Differ. 10: 899-904.

Wilkie, G.S., Dickson, K.S., and Gray, N.K. 2003. Regulation of mRNA translation by $5^{\prime}$ - and $3^{\prime}$-UTR-binding factors. Trends Biochem. Sci. 28: 182-188.

Yaman, I., Fernandez, J., Liu, H., Caprara, M., Komar, A.A., Koromilas, A.E., Zhou, L., Snider, M.D., Scheuner, D., Kaufman, R.J., et al. 2003. The zipper model of translational control: A small upstream ORF is the switch that controls structural remodeling of an mRNA leader. Cell 113: 519-531.

Yueh, A. and Schneider, R.J. 1996. Selective translation initia- tion by ribosome jumping in adenovirus-infected and heatshocked cells. Genes \& Dev. 10: 1557-1567.

Zhang, Z. and Dietrich, F.S. 2005. Identification and characterization of upstream open reading frames (uORF) in the 5' untranslated regions (UTR) of genes in Saccharomyces cerevisiae. Curr. Genet. 48: 77-87. 


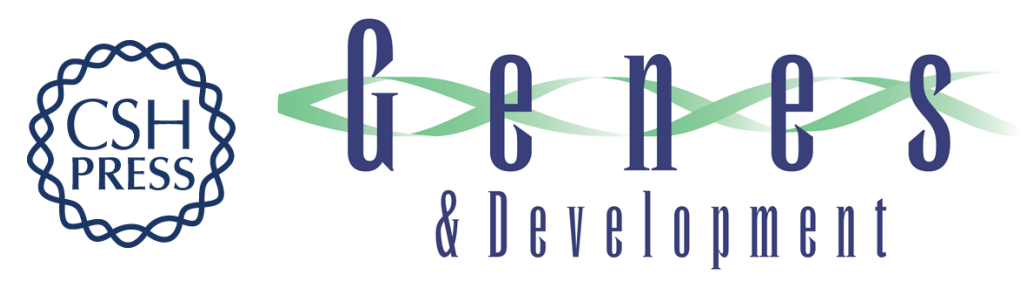

\title{
Downstream control of upstream open reading frames
}

\author{
Matthew S. Sachs and Adam P. Geballe
}

Genes Dev. 2006, 20:

Access the most recent version at doi:10.1101/gad.1427006

\section{Related Content Derepression of the Her-2 uORF is mediated by a novel post-transcriptional control mechanism in cancer cells \\ Anuradha Mehta, Christopher R. Trotta and Stuart W. Peltz \\ Genes Dev. UNKNOWN , 2006 20: 939-953 \\ References This article cites 57 articles, 25 of which can be accessed free at: \\ http://genesdev.cshlp.org/content/20/8/915.full.html\#ref-list-1 \\ Articles cited in: \\ http://genesdev.cshlp.org/content/20/8/915.full.htmI\#related-urls \\ License \\ Email Alerting Receive free email alerts when new articles cite this article - sign up in the box at the top \\ Service right corner of the article or click here.}

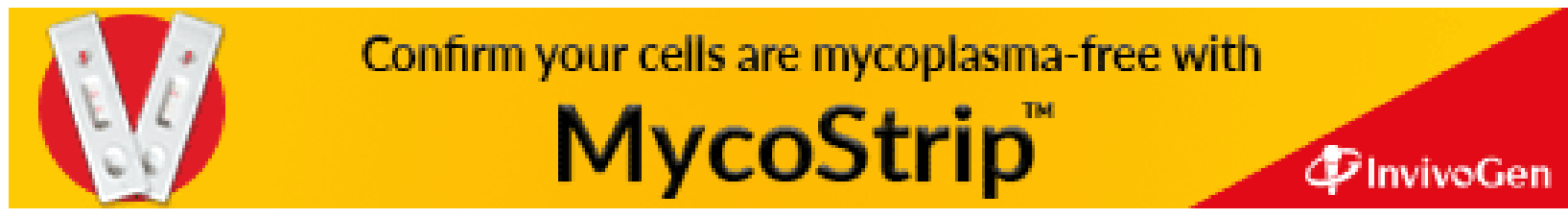

